\title{
openheart Evaluation of low gradient severe aortic stenosis: should we change our outlook in the analysis of clinical data?
}

Ivan Corazza (i) , Margherita Zecchi, Romano Zannoli

To cite: Corazza I, Zecchi M, Zannoli R. Evaluation of low gradient severe aortic stenosis: should we change our outlook in the analysis of clinical data? Open Heart 2021;8:e001746. doi:10.1136/

openhrt-2021-001746

Accepted 22 September 2021

Check for updates

(C) Author(s) (or their employer(s)) 2021. Re-use permitted under CC BY-NC. No commercial re-use. See rights and permissions. Published by BMJ.

Department of Experimental Diagnostic and Specialty Medicine, Alma Mater Studiorum University of Bologna, Bologna, Italy

Correspondence to Dr Ivan Corazza; ivan.corazza@ unibo.it

\section{ABSTRACT}

Nowadays, technological progress has equipped clinicians with new useful devices for the collection, analysis and presentation of data. As a consequence, many diseases and pathological conditions have been studied in a more detailed way, sometimes with remarkable results. In fact, they are not always validated by the old physiological models. In this respect, we present the case of low gradient severe aortic stenosis, a condition characterised by a small aortic valve area and a low-pressure gradient. According to the mathematical and physical assumptions these readings are contradictory whereas the Dopplerechocardiography shows clearly the existence of such a situation. In this work, we have described the physiological base of this phenomenon and discussed the limitations of the technology used. In this work, we are going to analyse some conditions commonly observed in daily clinical practice in order to prompt a critical outlook in both clinicians and technicians about the instrumentations used and the methods applied.

\section{INTRODUCTION}

In recent years, in the medical field, there has been a rapid technological evolution and a consequent abundance of clinical data available to the specialist. However, these new devices are based on traditional scientific and physiological principles. Therefore, real innovation is represented by the development of increasingly powerful and complex algorithms to improve the quality of the presentation of the results. On the one hand, the new techniques provide a better view of patients' physiological and pathological conditions, but on the other hand, some inconsistencies arouse, between diagnosis and physical models. An example that attracted our attention was the publication of articles demonstrating the existence of severe aortic stenosis (AS) with a low-pressure gradient.

In the last years, several papers were published stating the clinical evidence of an important proportion of patients with AS and 'low-gradient' AS: that is, a small aortic valve area (AVA $<1.0 \mathrm{~cm}^{2}$ ) consistent with severe AS but a low mean trans-valvular gradient $(\Delta P$,
$\Delta P<40 \mathrm{~mm} \mathrm{Hg})$ consistent with non-severe AS. ${ }^{1-3}$ These works aim to better diagnose the pathologies related to aortic valves and so to improve patient classification and therapy administration. Even if this is a crucial goal, it would be interesting to better investigate the physical model undergoing the human physiology and the medical instrumentations. Moreover, the increasing complexity of statistical analysis of small and big clinical data tends to push final results away from the clinical procedure and instrumental apparatus used to make the measurements, diverting the researchers' eyes from the critical evaluation of the reliability of their findings.

Following this awareness, we decided to focus our attention on the theoretical and procedural aspects involved in the AS evaluation.

\section{State of the art}

\section{Mathematical model}

To study what happens at the stenosis level, blood is considered an ideal fluid and Bernoulli theorem (1) and the continuity equation for an ideal liquid (Castelli Law) (2) are used to quantify the pressure gradient (figure 1) ${ }^{45}$ By considering the section of the stenosis and a section inside the ventricle, we can state that:

$$
\begin{gathered}
P_{S}+\rho g H_{S}+\frac{1}{2} \rho v_{S}^{2}=P_{V}+\rho g H_{V}+\frac{1}{2} \rho v_{V}^{2} \\
Q=\mathrm{AVA} \cdot v_{S}=A_{V} \cdot v_{V}
\end{gathered}
$$

Where:

$P_{S}=$ pressure at the stenosis level.

$H_{\mathrm{s}}=$ height of the stenosis from the ground.

$\rho=$ blood density.

$v=$ blood velocity in the stenosis.

$g=$ gravitational acceleration $\left(9.8 \mathrm{~m} / \mathrm{s}^{2}\right)$.

$P_{\mathrm{V}}=$ pressure in the ventricle.

$H_{\mathrm{V}}=$ height of the ventricle from the ground. $v_{\mathrm{V}}=$ blood velocity in the ventricle.

$Q=$ cardiac output.

AVA=area of the stenosis. 


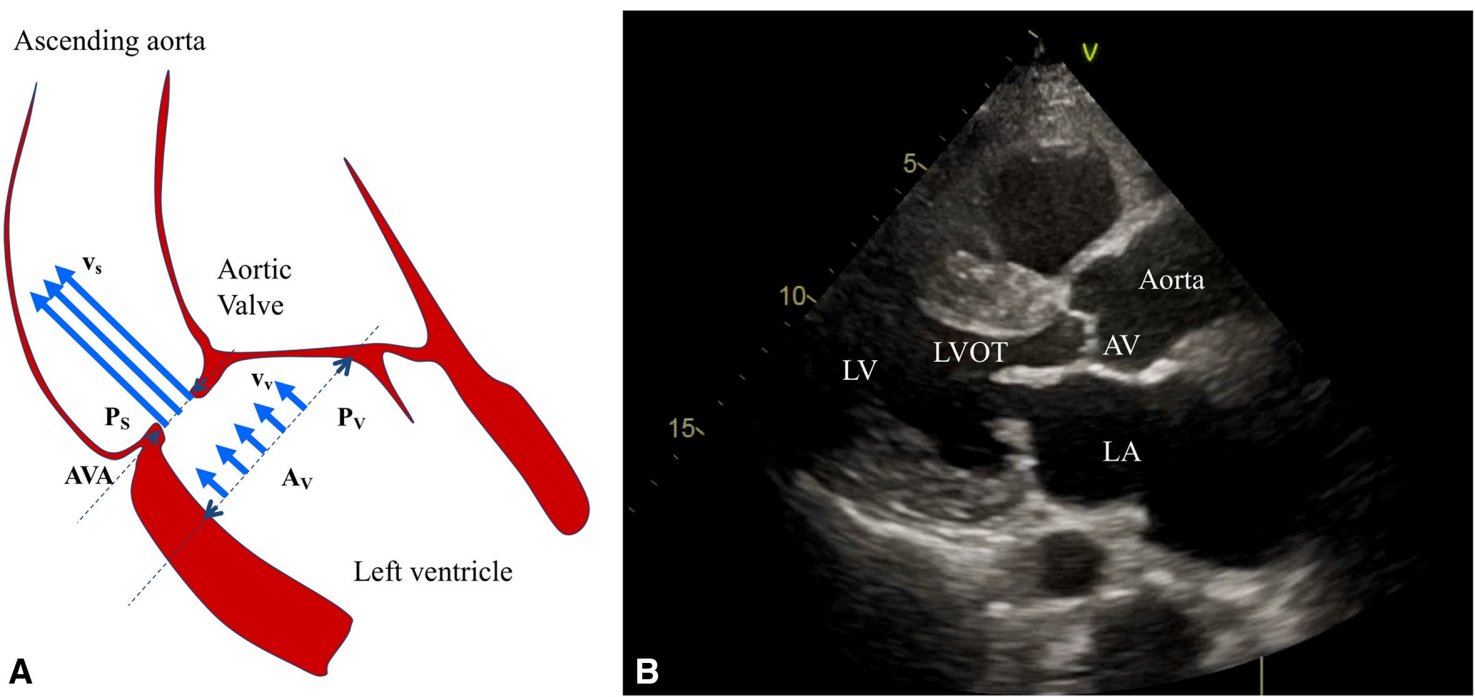

Figure 1 (A) Schematic and ideal representation of left ventricle, aortic valve and aorta, considering the blood an ideal fluid. It is possible to notice that the blood speed is constant across each considered section. $A_{v}$, ventricular area; AVA, stenosis area; $P_{S}$, stenosis pressure; $P_{v}$, ventricular pressure; $v_{s}$, blood velocity in the stenosis; $v_{v}$, velocity on the ventricle. (B) Parasternal long axis view obtained with standard echography. AV, aortic valve; LA, left atrium; LV, left ventricle; LVOT, left ventricle outflow tract.

$A_{\mathrm{V}}=$ area of the ventricular section in the outflow tract.

Making the hypothesis that $H_{S}=H_{V}$ and $A_{V}>$ AVA (with the consequence that $v_{\mathrm{s}}>v_{\mathrm{V}}$ ) and combining the two equations, we obtain the Gorlin formula ${ }^{67}$ that allows calculating the stenosis area by measuring the pressure gradient between ventricle and valve and the cardiac output:

$$
\mathrm{AVA}=\sqrt{\frac{1}{2} \rho \frac{Q^{2}}{\Delta P}}=Q \sqrt{\frac{\rho}{2 \Delta P}}
$$

If we want to calculate the pressure gradient, by combining the Gorlin with the continuity equation for an ideal fluid we obtain:

$$
\Delta P=\frac{\rho}{2} v_{S}^{2}
$$

So, knowing the blood density, by measuring the blood velocity inside the stenosis, it is possible to calculate the pressure gradient. Moreover, expressing the speed in metres per second $(\mathrm{m} / \mathrm{s})$ and the pressure in millimetres of mercury $(1 \mathrm{~mm} \mathrm{Hg}=133 \mathrm{~Pa})$, the above formula becomes:

$$
\Delta P \cong 4 v_{s}^{2}
$$

Since the flow is pulsatile, all the values considered for calculation of AVA are the mean ones along with the ventricular ejection phase. The estimation of aortic valve insufficiency in daily clinical practice is commonly made with echo Doppler or cardiac catheterisation.

\section{Echo Doppler approach}

By using a continuous wave Doppler (CWD) flowmeter, ${ }^{8}$ it is possible to measure the blood speed inside the stenosis section and then calculate the pressure gradient. Since the flow is pulsatile, the mean velocity during ejection is calculated.

To measure the stenosis area, the diameter of the section of the ventricular outflow tract $\left(D_{V}\right)$ is measured with echography in mid-systole and the ventricular blood speed with a pulsed wave (PW) Doppler device. ${ }^{8}$ By applying the continuity equation (2), AVA can be easily calculated (6). It is important to notice that $A_{V}$ is hypothesised circular (7) and all the considered values are mediated along with different beats.

$$
\begin{aligned}
& \text { AVA }=\frac{A_{V} \cdot v_{V}}{v_{s}} \\
& A_{V}=\pi\left(\frac{D_{V}}{2}\right)^{2}
\end{aligned}
$$

\section{Cardiac catheterisation}

The trans-valvular pressure gradient across the aortic valve is measured by the use of a catheter in the left ventricle (LV) and another in the proximal aorta. ${ }^{7}$ This approach allows to directly measure the pressure gradient across the valve but its application is limited since the procedure is invasive and more expensive. ${ }^{910}$

\section{Other techniques}

Actually, with cardiac magnetic resonance ${ }^{11-13}$ it is possible to measure the blood velocity and to calculate through the above relation (5) the pressure gradient.

\section{Consequences}

If the above model is correct and the measurements reliable, it is quite obvious that if the AVA is small (severe stenosis), the blood speed inside the stenosis should be much higher than the one in the ventricle and the pressure gradient should be high.

If this thing does not happen, something in the mathematical and physiological models or in the measurement procedure is wrong.

In this work, we will try to deepen the problem so as to highlight the weakness of the applied model and the standard clinical measurements issues. 


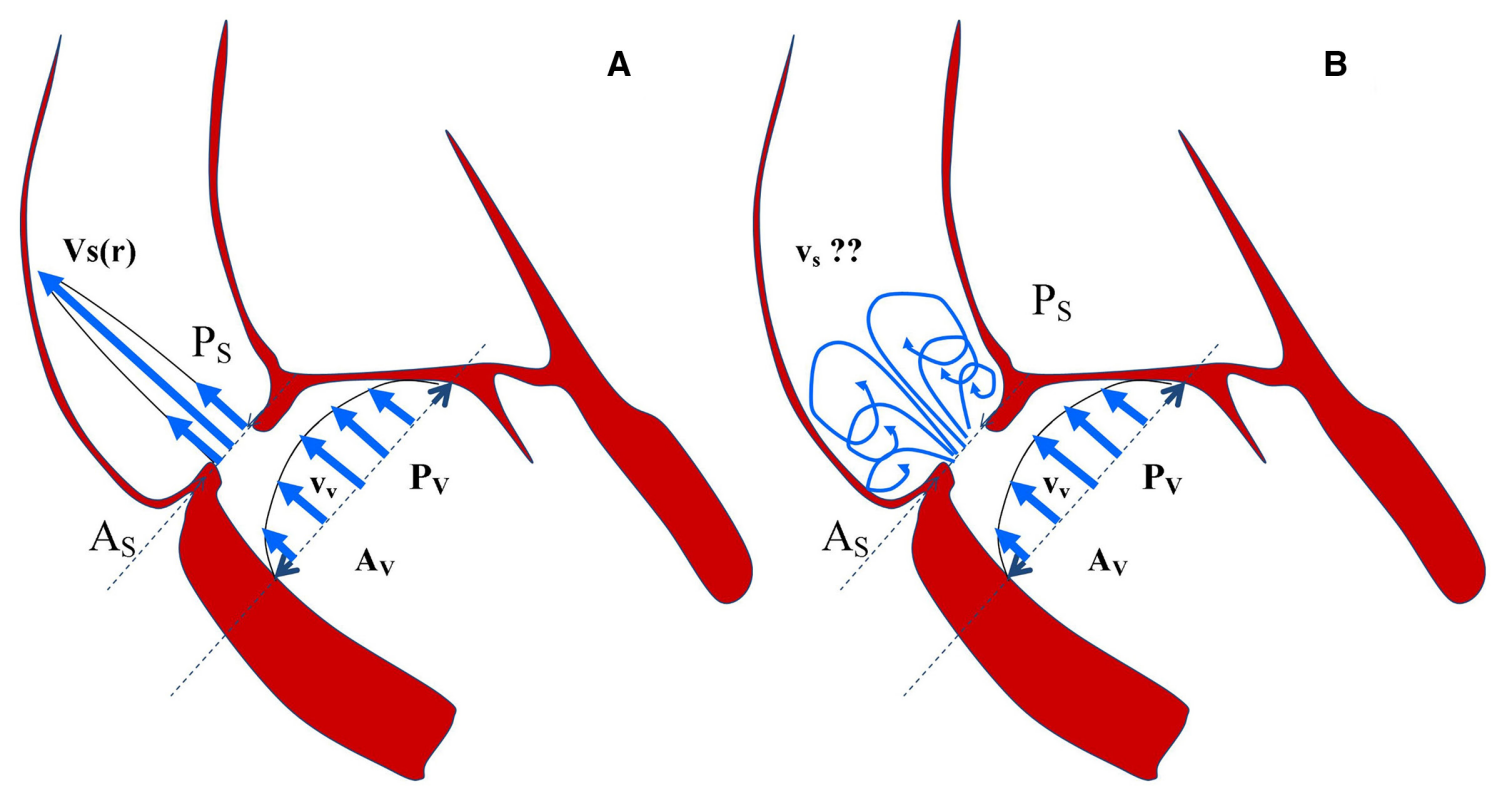

Figure 2 (A) Laminar blood flow in ventricle and across the valve; (B) laminar flow in ventricle and turbulent flow in aorta. $A_{V}$, ventricular area; $P_{s}$, stenosis pressure; $P_{v}$, ventricular pressure; $v_{s}$, blood velocity in the stenosis; Vs(r), blood velocity in the stenosis as function of the distance from the valve's wall $r ; v_{v}$, velocity on the ventricle; As, stenosis area.

\section{CRITICAL EVALUATION OF THE STANDARD METHODS}

To better understand the weakness of the above explanations, we will try to be schematic and to divide the following paragraph with the same structure as the previous one.

\section{Mathematical model}

The accepted mathematical model undergoing the clinical approach to evaluate AS assumes that the blood behaves as an ideal fluid, moving at a constant speed both across sections and in time. This is a valid hypothesis for short vessels with big diameters: in these cases, the vascular resistance can be considered negligible and the fluid ideal (viscosity equal to zero). Despite the small length and the big diameter of the ventricle, this model cannot be applied to AS owing to the small diameter of the stenosis, not compatible with the hypothesis.

Moreover, since the blood viscosity is not zero $(\sim 4 \mathrm{mPa}$ s) the blood is a real fluid and the flow should present a parabolic profile (laminar flow), both in ventricle and inside the AS (figure 2A), and the speed is impulsive, following the ventricular contractions.

Let us try to analyse what really happens in the stenosis and in the ventricle.

As a consequence, the blood speed is not constant along the stenosis section, but it presents a maximum value at the centre and a nearly zero value near the lateral walls.

Moreover, since the speed just outside the stenosis is high (and becomes higher if AVA is smaller) it is highly probable that the laminar flow switches in turbulent inside ascending aorta (figure 2B). The ideal fluid model is really far from this real situation and the common Doppler technique to measure velocity across the stenosis can lose its reliability if the velocity is not measured in the right section (see the next paragraphs).
According to the ideal fluid model, the speed of the fluid in the ventricle and across the section is the same. If the ventricular flow is laminar, blood speed has different values across the stenosis section. And this is the first problem.

Another important aspect to be considered is related to the direction of the velocity vector. As stated by the ideal fluid model, the speed is orthogonal both to the valve and to the ventricular sections.

If the ventricle presents morphological alterations due to cardiovascular pathologies (hypertrophy, dilation, ischaemia, etc), the speed vector is certainly not perpendicular to the section, the profile is not parabolic and the blood trajectory is different (figure 3).

When AVA evaluation is made through the continuity equation, if the ventricular section is not circular (as hypothesized in the model, see the Echo Doppler approach subsection in the Introduction section) but tends to become elliptical near the valve, the measurement of $A_{V}$ is mandatory to obtain reliable results. If the ventricle is dilated or hypertrophied, this aspect should not be neglected.

As a consequence, the model could become unreliable in some serious pathological conditions, leading to wrong results.

Since the relationship between blood speed at the stenosis level and pressure gradient is quadratic, even if the ideal model can be applied, a little mistake in velocity can lead to a big mistake in terms of the gradient.

\section{Echo Doppler approach}

To properly understand the limitation of the approach, it is important to underline some basic concepts:

1. To measure blood velocity in the stenosis, a standard CWD flowmeter is commonly used. This technique is 


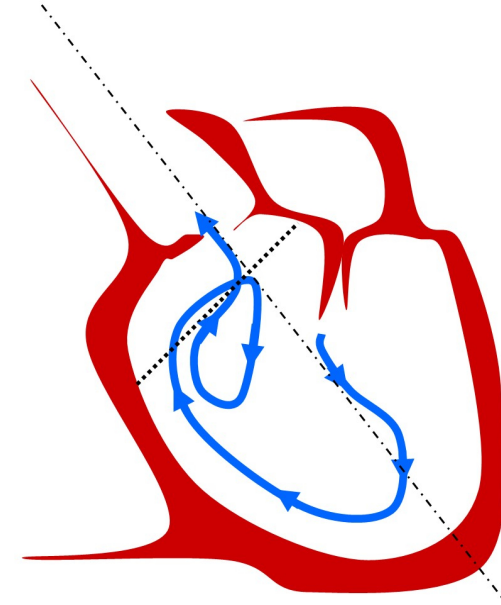

A

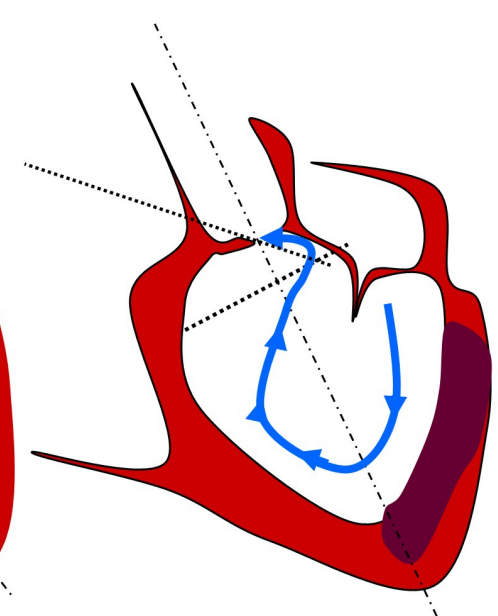

B

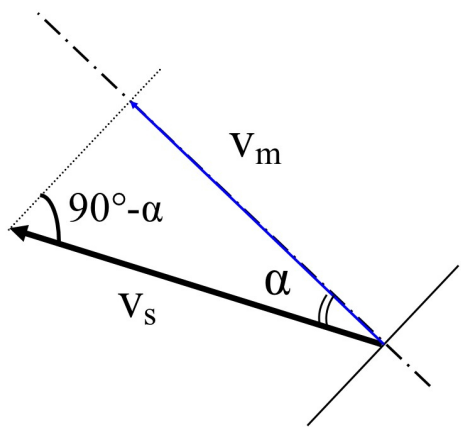

C

Figure 3 Example of blood flow lines in case of a (A) dilated ventricle and $(B)$ an ischaemic one (the darkest wall area represents the ischaemic part of the ventricle). The blood velocity is not the same along each section and not always perpendicular to it: (C) example of speed not parallel to ultrasounds propagation axis. $v_{s}$, mean velocity across the stenosis; $v_{m}$, measured mean velocity; $\alpha$, angle between US propagation axis and velocity.

based on waves frequency change due to reflection or diffusion by a moving object. This frequency difference is proportional to the component of the speed vector parallel to the ultrasounds propagation axis. Typically, the US transducer is placed so as to have an axis perpendicular to valvular surface but if the blood flow has a different direction (eg, figure 3), the measured speed is underestimated and so is the gradient (4). Moreover, the CWD approach cannot permit to localise the measurement and the final result is the superimposition of the speed of all blood particles invested by the US bundle. In this case, the speed can be underestimated depending on the geometrical distribution of the laminar flow and the presence of turbulence.

2. The measurement of velocity in the ventricle is done with PW Doppler: this approach allows to avoid localisation problems but the reliability of the final speed value depends always on the position of the measurement point compared with the ventricular walls. If the blood flows into the ventricle in an abnormal way (due, eg, to a hypertrophic cardiomyopathy), the ventricular blood speed can be completely wrong. Moreover, the presence of an obstruction in the left ventricle outflow tract, since $A \mathrm{~V}$ decreases, generates an increase in $v \mathrm{~V}$ and, consequently, in vs. It is fundamental to correctly measure $v \mathrm{~V}$ and $A \mathrm{~V}$ in the same outflow tract $\left(A_{V} \cdot v_{V}\right.$ is constant) otherwise the 'continuity' hypothesis cannot be applied.

A numerical example of what can happen if blood velocity is not parallel to the US propagation axis is proposed (see figure 3C):

\section{Measured mean values:}

$v_{m}=3.0 \mathrm{~m} / \mathrm{s}$.

$v_{V}=1.0 \mathrm{~m} / \mathrm{s}$.
$D_{V}=2.4 \mathrm{~cm}$.

Calculated values:

$\Delta P=36 \mathrm{~mm} \mathrm{Hg}$.

$R_{V}=1.2 \mathrm{~cm}$.

$A_{\mathrm{V}}=4.52 \mathrm{~cm}^{2}$.

$\mathrm{AVA}=1.5 \mathrm{~cm}^{2}$.

By analysing these results, the AS could be classified mild/moderate.

What happens if the $v_{m}$ is not the full velocity module but only a component? Let us consider the case of $\alpha=30^{\circ}$.

The real total velocity module $\left(v_{s}\right)$ results $3.46 \mathrm{~m} / \mathrm{s}$ and $\mathrm{AVA}=1.3 \mathrm{~cm}^{2}$ with a pressure gradient of $48 \mathrm{~mm} \mathrm{Hg}$ and the correct final diagnosis should be of a moderate stenosis. If the angle is $60^{\circ}, v$ is $6 \mathrm{~m} / \mathrm{s}$ and AVA results 0.75 $\mathrm{cm}^{2}$ and $\Delta P=144 \mathrm{~mm} \mathrm{Hg}$ with the consequence of a real severe stenosis. In this last case, if the angle is not considered in the calculations, the severity of the pathology is underestimated with possible serious consequences.

Moreover, some possible mistakes in $A_{V}$ and/or $v_{V}$ estimation should be considered since they can alter the final results significantly. ${ }^{14}$

Last but not least, measurements errors due to the experimental apparatus and practical procedure must be considered. The axial resolution of a standard system corresponds to the wavelength of the ultrasounds. A transducer with a $3 \mathrm{MHz}$ frequency (considering the US velocity about $1400 \mathrm{~m} / \mathrm{s}$ ) has an intrinsic resolution of $0.5 \mathrm{~mm}$. The error doubles measuring diameters $(1 \mathrm{~mm})$ and becomes higher if surfaces or volumes are estimated.

If we add to these considerations the errors due to the ventricular geometrical model ${ }^{15}$ and the difficulties of the operator to properly define the ventricle internal wall surface, it is clear how the measurement of ventricle diameter can negatively affect the calculation of the AVA (2). 
Referring to the above example, here it is what happens in the best condition $\left(\alpha=0^{\circ}\right)$ only considering the errors on the $\mathrm{LV}$ diameter.

$v_{m}=3.0 \mathrm{~m} / \mathrm{s}$

$v_{V}=1.0 \mathrm{~m} / \mathrm{s}$

$D_{V}=(2.4 \pm 0.1) \mathrm{cm}$

$\Delta \mathrm{p}=36 \mathrm{~mm} \mathrm{Hg}$

$\mathrm{R}_{\mathrm{v}}=(1.20 \pm 0.05) \mathrm{cm}$.

$A_{V}=(4.5 \pm 0.4) \mathrm{cm}^{2}$.

AVA $=(1.50 \pm 0.13) \mathrm{cm}^{2}$.

The axial resolution of the echocardiograph alone is responsible for an error of $10 \%$ on the final AVA value (without considering the errors on the speed and those due to the procedure).

Most of the research papers, guidelines and randomised trials on echography and Doppler assessment do not consider the resolution of the technological apparatus. Usually, the instrumentation is considered fully reliable and the only uncertainties are the ones due to the procedural approach and the operators. Often these errors tend to be reduced only by statistical methods, increasing the number of cases, even if this is not a wise procedure.

\section{Cardiac catheterisation}

The standard approach provides the measurement of the pressure decay across the valve, the cardiac output and then, using the Gorlin formula (3) the valve area can be calculated. The results reliability depends on the accuracy of the physiological model and on the pressure measurements. In particular, a good measure requires the pressure to be measured or just outside the valve area. Actually, if the pressure transducer is placed far from the valve section, due to the enlargement of the aorta, the pressure can be overestimated ${ }^{16} 17$ with an underestimation of the pressure gradient and a consequent error in the valve area calculation.

Similar problems can arise if the flow is turbulent and the pressure is not constant along the aorta axis.

\section{Other techniques}

CT allows a greater spatial resolution and a better definition of the geometry of the valve and of the stenosis. Nevertheless, it does not provide any quantification of the haemodynamic parameters. Moreover, CT requires the patients to be highly exposed to X-ray exposure and the procedure is longer and more expensive than the echography approach. ${ }^{18}$

On the contrary, MRI provides a complete evaluation making it possible to estimate both geometry and haemodynamic without risks deriving from ionising radiation exposure. The use of this technique is constantly increasing ${ }^{19}$ and gives better results in terms of concordance with the mechanical model ${ }^{20}{ }^{21}$ but it presents the same limitations of CT since both are very expensive and cannot be performed at the bedside.

\section{DISCUSSION}

Our considerations are not intended to be a denunciation of wrong or inappropriate behaviour, but represent a starting point that can help clinicians, on the one hand, and technicians (us, too), on the other, to improve our way of working.

The main objective of our paper is not to simply highlight the limitations of a physiological model and of the instrumentation used to evaluate the behaviour of the aortic valve: lots of papers have been published in the last decades about this topic ${ }^{152-26}$ but nothing has changed in the daily clinical practice and the algorithms used to make the diagnosis are the same. Some corrections have been proposed and implemented ${ }^{1827-32}$ but the underlying uncertainty due to the model's approximations and technological limitations are still present. As a consequence, some paradoxical phenomena, like low-gradient severe stenosis are still detected.

Lots of papers have been published to highlight these last topics. A quick PubMed search of the keywords "lowgradient severe aortic stenosis" reveals about 400 papers published from 1996 till now (2021). So, the argument is still of great interest and a common solution to overcome the paradox does not exist.

Probably the greatest limitation of our work lies in simplification: we voluntarily treated the subject in a very simple and simplified way, with easy-to-understand numerical examples, without resorting to complex mathematical models: our goal was certainly not to solve the paradox of low-gradient AS, but to provide an idea of discussion.

We would like to feed the thoughts of clinicians and technicians daily working with performing and powerful instrumentation and well-defined, but sometimes unknown, physiological models.

It is undeniable that, for those who, like us, deal with medical instrumentation, it is essential to improve the performance and usability of the equipment, refining the quality of the results provided. For example, a beautiful radiological image, filtered and optimised through increasingly advanced imaging algorithms, certainly represents a goal to aim for, but it risks overshadowing the method with which this image was constructed.

This discrepancy between what really is and what appears, in most cases, does not in any way alter the diagnostic chain, the therapeutic process and the final outcome, because the expert clinician knows how to interpret the data provided by the machines by comparing them with other clinical parameters and with the anamnesis.

In this scenario, clinicians' experience plays a major role since a deep understanding of the limits and the technical specification of the equipment is imperative for a correct diagnosis. At the same time, it is crucial that both technicians and commercial agents highlight the characteristics of a specific product. This acquires a significantly greater importance in a historical period in which 
international guidelines are usually followed step by step, without considering the intrinsic limits of the procedure and the error of the diagnostic instrumentation used in that specific case. As a matter of fact, if the ultrasound examination, planned and carried out according to the guidelines, indicates severe stenosis, then the patient must undergo valve replacement.

This lack of knowledge is partially due to the way by which both clinicians and technicians try to keep themselves up to date. As proof, usually, the technological equipment used in hospitals is presented and discussed in technical journals, not always considered by clinicians. On the other hand, technicians are not always well aware of the complexity of a certain pathological condition from a medical point of view.

The example of this article is clear: the diagnosis of severe AS, in the face of a low-pressure gradient across the valve. The physical and physiological models underlying the valvular diameter measurement procedures cannot allow such a result. At this point, either the result or the method or both are wrong. Probably neither the results nor the methods are absolutely incorrect, anyway there are limits on both fronts: the method is based on physical assumptions and hypotheses that are not always applicable to physiology and the result is provided through the use of diagnostic tools with important intrinsic limitations.

We do not have a single and certain answer to the proposed problem and we do not have the presumption and the ability to furnish definitive answers. However, we questioned whether these evidences are reliable or not and we would like this doubt to belong to all those involved in investigating aortic insufficiency.

To sum up, in our opinion, the limitations of clinical procedures and the equipment in use should be taught to the professionals that deal with them in their daily work. This would allow broadening a patient's diagnostic path with more instrumental investigations when necessary.

Contributors Conceptualisation: IC, RZ. Formal analysis and investigation: IC, MZ. Writing - original draft preparation: IC, MZ. Writing - review and editing: IC, MZ.

Funding The authors have not declared a specific grant for this research from any funding agency in the public, commercial or not-for-profit sectors.

Competing interests None declared.

Patient consent for publication Not applicable.

Provenance and peer review Not commissioned; externally peer reviewed.

Data availability statement There are no data in this work.

Open access This is an open access article distributed in accordance with the Creative Commons Attribution Non Commercial (CC BY-NC 4.0) license, which permits others to distribute, remix, adapt, build upon this work non-commercially, and license their derivative works on different terms, provided the original work is properly cited, appropriate credit is given, any changes made indicated, and the use is non-commercial. See: http://creativecommons.org/licenses/by-nc/4.0/.

ORCID iD

Ivan Corazza http://orcid.org/0000-0003-2078-1978

\section{REFERENCES}

1 Clavel M-A, Magne J, Pibarot P. Low-gradient aortic stenosis. Eur Heart J 2016;37:2645-57.
2 Clavel M-A, Pibarot P, Dumesnil JG. Paradoxical low flow aortic valve stenosis: incidence, evaluation, and clinical significance. Curr Cardiol Rep 2014;16:431.

3 Pibarot P, Dumesnil JG, Low-Flow P. Paradoxical low-flow, lowgradient aortic stenosis: new evidence, more questions. Circulation 2013;128:1729-32

4 Carabello BA, Paulus WJ. Aortic stenosis. Lancet 2009;373:956-66.

5 Okura H, Yoshida K, Hozumi T, et al. Planimetry and transthoracic two-dimensional echocardiography in noninvasive assessment of aortic valve area in patients with valvular aortic stenosis. J Am Coll Cardiol 1997;30:753-9.

6 Gorlin R, Gorlin SG. Hydraulic formula for calculation of the area of the stenotic mitral valve, other cardiac valves, and central circulatory shunts. I. Am Heart J 1951;41:1-29.

7 Joseph J, Naqvi SY, Giri J, et al. Aortic stenosis: pathophysiology, diagnosis, and therapy. Am J Med 2017;130:253-63.

8 Bashford GR. Ultrasonic measurement of blood flow velocity and applications for cardiovascular assessments. In: Patel VB, Preedy VR, eds. Biomarkers in cardiovascular disease. Dordrecht: Springer Netherlands, 2015: 1-31.

9 Massa L, Vitrella G, Zecchin M. [Indications for cardiology consultation and management of cardiac patients who will undergo surgical or endoscopic procedures: the proposal of the University Hospital of Trieste, Italy]. G Ital Cardiol 2010;2006:590-8.

10 Wilson W, Taubert KA, Gewitz M, et al. Prevention of infective endocarditis: guidelines from the American heart association: a guideline from the American heart association rheumatic fever, endocarditis, and Kawasaki disease Committee, Council on cardiovascular disease in the young, and the Council on clinical cardiology, Council on cardiovascular surgery and anesthesia, and the quality of care and outcomes research interdisciplinary Working group. Circulation 2007;116:1736-54.

11 Chai P, Mohiaddin R. How we perform cardiovascular magnetic resonance flow assessment using phase-contrast velocity mapping. J Cardiovasc Magn Reson 2005;7:705-16.

12 Wymer DT, Patel KP, Burke WF, et al. Phase-contrast MRI: physics, techniques, and clinical applications. Radiographics 2020;40:122-40.

13 Spampinato RA, Jahnke C, Paetsch I, et al. Quantification of aortic valve regurgitation by pulsed Doppler examination of the left subclavian artery velocity contour: a validation study with cardiovascular magnetic resonance imaging. J Am Soc Echocardiogr 2018;31:42-51

14 Chin CWL, Khaw HJ, Luo E, et al. Echocardiography underestimates stroke volume and aortic valve area: implications for patients with small-area low-gradient aortic stenosis. Can J Cardiol 2014;30:1064-72.

15 Gilja OH, Hausken T, Berstad A, et al. Measurements of organ volume by ultrasonography. Proc Inst Mech Eng H 1999;213:247-59.

16 Voelker W, Reul H, Stelzer T, et al. Pressure recovery in aortic stenosis: an in vitro study in a pulsatile flow model. J Am Coll Cardiol 1992;20:1585-93.

17 Laskey WK, Kussmaul WG. Pressure recovery in aortic valve stenosis. Circulation 1994;89:116-21.

18 Saikrishnan N, Kumar G, Sawaya FJ, et al. Accurate assessment of aortic stenosis: a review of diagnostic modalities and hemodynamics. Circulation 2014;129:244-53.

19 Gulsin GS, Singh A, McCann GP. Cardiovascular magnetic resonance in the evaluation of heart valve disease. BMC Med Imaging 2017;17:67.

20 Adriaans BP, Westenberg JJM, van Cauteren YJM, et al. Clinical assessment of aortic valve stenosis: comparison between 4D flow $\mathrm{MRI}$ and transthoracic echocardiography. J Magn Reson Imaging 2020;51:472-80.

21 Garcia J, Barker AJ, Markl M. The role of imaging of flow patterns by 4D flow MRI in aortic stenosis. JACC Cardiovasc Imaging 2019;12:252-66.

22 Burwash IG, Thomas DD, Sadahiro M, et al. Dependence of Gorlin formula and continuity equation valve areas on transvalvular volume flow rate in valvular aortic stenosis. Circulation 1994;89:827-35.

23 Chambers JB, Sprigings DC, Cochrane T, et al. Continuity equation and Gorlin formula compared with directly observed orifice area in native and prosthetic aortic valves. Br Heart J 1992;67:193-9.

24 Rifkin RD. Physiological basis of flow dependence of Gorlin formula valve area in aortic stenosis: analysis using an hydraulic model of pulsatile flow. J Heart Valve Dis 2000;9:740-51.

25 Tardif JC, Rodrigues AG, Hardy JF, et al. Simultaneous determination of aortic valve area by the Gorlin formula and by transesophageal echocardiography under different transvalvular flow conditions. Evidence that anatomic aortic valve area does not change 
with variations in flow in aortic stenosis. J Am Coll Cardiol 1997;29:1296-302.

26 Wippermann CF, Schranz D. Overestimation of valve area by the Gorlin formula. J Am Coll Cardiol 1992;19:234-5.

27 Baumgartner $\mathrm{H}$, Hung J, Bermejo J, et al. Recommendations on the echocardiographic assessment of aortic valve stenosis: a focused update from the European Association of Cardiovascular Imaging and the American Society of Echocardiography. Eur Heart $J$ Cardiovasc Imaging 2017;18:254-75.

28 Combining Automatic Angle Correction and 3-D Tracking Doppler for the Assessment of Aortic Stenosis Severity - PubMed. Available: https:// pubmed.ncbi.nlm.nih.gov/31180850/ [Accessed 12 May 2021].
29 Cutting WB, Bavry AA. The evolving approach to the evaluation of low-gradient aortic stenosis. Cardiovasc Revasc Med 2019;20:197-201.

30 Hakki AH, Iskandrian AS, Bemis CE, et al. A simplified valve formula for the calculation of stenotic cardiac valve areas. Circulation 1981;63:1050-5.

31 Hare TW, Hakki AH, Iskandrian AS. Comparison between the Gorlin formula and a simplified formula to measure the severity of pulmonic stenosis. Cathet Cardiovasc Diagn 1983;9:353-6.

32 Kwon J-M, Lee SY, Jeon K-H, et al. Deep learning-based algorithm for detecting aortic stenosis using electrocardiography. J Am Heart Assoc 2020;9:e014717. 